\title{
Les enseignements du VIH: combattre l'hépatite virale
}

\author{
Philip Bruggmann ${ }^{a}$, Bettina Maeschlib, Gert Printzenc \\ aPD Dr med., directeur de la Stratégie nationale de lutte contre l'hépatite; \\ ${ }^{b}$ lic. phil. I, responsable de la communication, Stratégie nationale de lutte contre I'hépatite; \\ 'Dr med., membre du Comité central de la FMH et du réseau de la Stratégie nationale de lutte contre I’hépatite
}

Plus de 100000 personnes en Suisse vivent avec une hépatite chronique. Cette maladie est la cause la plus fréquente de cancer du foie et de transplantation hépatique. Pourtant, on n'en parle encore guère dans notre pays. Un réseau de plus de 80 personnalités veut que cela change, et travaille d'arrache-pied à la mise au point d'une stratégie de lutte contre l'hépatite dans le but d'éliminer l'hépatite virale en Suisse à l'horizon 2030.

Depuis quelques années, monsieur S., 52 ans, est de plus en plus gêné dans son activité de comptable par une fatigue chronique et des difficultés de concentration. Pour cette raison, son médecin traitant le met régulièrement en arrêt maladie. La cause de la fatigue n'apparaît pas au premier abord. Des examens approfondis révèlent que monsieur S. souffre d'une hépatite $C$ chronique qu'il a probablement contractée lors d'une transfusion sanguine au début des années 80. D'autres étiologies fréquentes de sa fatigue ont pu être exclues. Le foie n'est cependant pas très affecté par l'infection.

Le cas de monsieur S. n'a rien d'exceptionnel. Plus de la moitié des personnes atteintes d'une hépatite virale ignorent qu'elles sont infectées. Souvent, la maladie ne se manifeste que plusieurs décennies après l'infection. Qui plus est, elle ne touche pas toujours le foie. C'est cette diversité des formes possibles d'hépatite virale, de l'hépatite $C$ en particulier, qui rend le diagnostic si difficile.

\section{Plus mortelle que le VIH/sida}

Selon l'Office fédéral de la santé publique (OFSP), la prévalence de l'hépatite $\mathrm{C}$ chronique (VHC) en Suisse s'élève à quelque 80000 cas, contre environ 24000 cas pour l'hépatite $\mathrm{B}(\mathrm{VHB})$, et les cas non recensés sont nombreux. Pour comparaison, la prévalence du VIH en Suisse est estimée à 15000 patients [1]. Depuis 2001, les décès annuels dus à l'hépatite $\mathrm{C}$ en Suisse sont plus nombreux que ceux dus au $\mathrm{VIH}^{1}$.

Malgré le fardeau que représente cette maladie et les graves conséquences possibles d'une hépatite chronique pour les patients, on ne parle guère de l'hépatite virale en Suisse. Une discrétion qui ne s'explique qu'en partie par l'évolution souvent peu spectaculaire de la

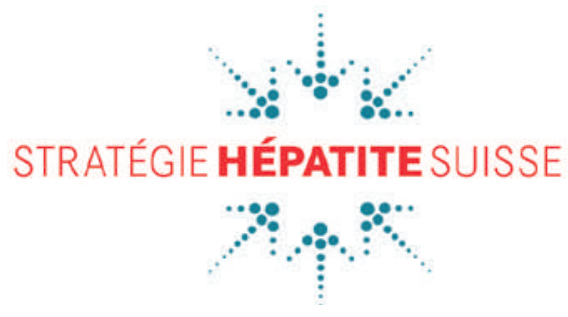

\section{La Stratégie nationale de lutte contre I’hépatite}

La Stratégie nationale de lutte contre I'hépatite a développé une vision et des objectifs en accord avec celle-ci: l'élimination de I'hépatite virale en Suisse à I'horizon 2030. C'est ainsi qu'elle entend réduire les infections chroniques de $30 \%$ dans les 5 ans à venir et les éliminer dans les 15 prochaines années. La survenue de nouveaux cas doit être réduite de $50 \%$ dans les 5 prochaines années et de $100 \%$ d'ici 15 ans.

Les 80 personnalités du réseau collaborent de manière bénévole à cette stratégie. Plus de 1000 heures de travail bénévole ont ainsi été accomplies en 2015.

maladie. Les conséquences d'une hépatite virale chronique ont souvent été sous-estimées par le passé. Les choses commencent cependant à bouger au niveau international et en Suisse.

La progression d'une hépatite infectieuse chronique est silencieuse. Un tiers des patients infectés par le VHC développent une cirrhose du foie, en général au terme d'une évolution de plusieurs années, voire de décennies. Il existe toujours plus d'éléments prouvant que l'hépatite $\mathrm{C}$ chronique est une importante maladie systémique qui peut se manifester dans d'autres organes du corps que le foie. L'hépatite $C$ est associée non seulement à une élévation de la mortalité hépatique, mais aussi de la mortalité générale chez les personnes concernées [2, 3]. Une infection à VHC peut entraîner une cryoglobulinémie. Celle-ci peut évoluer en vascu- 


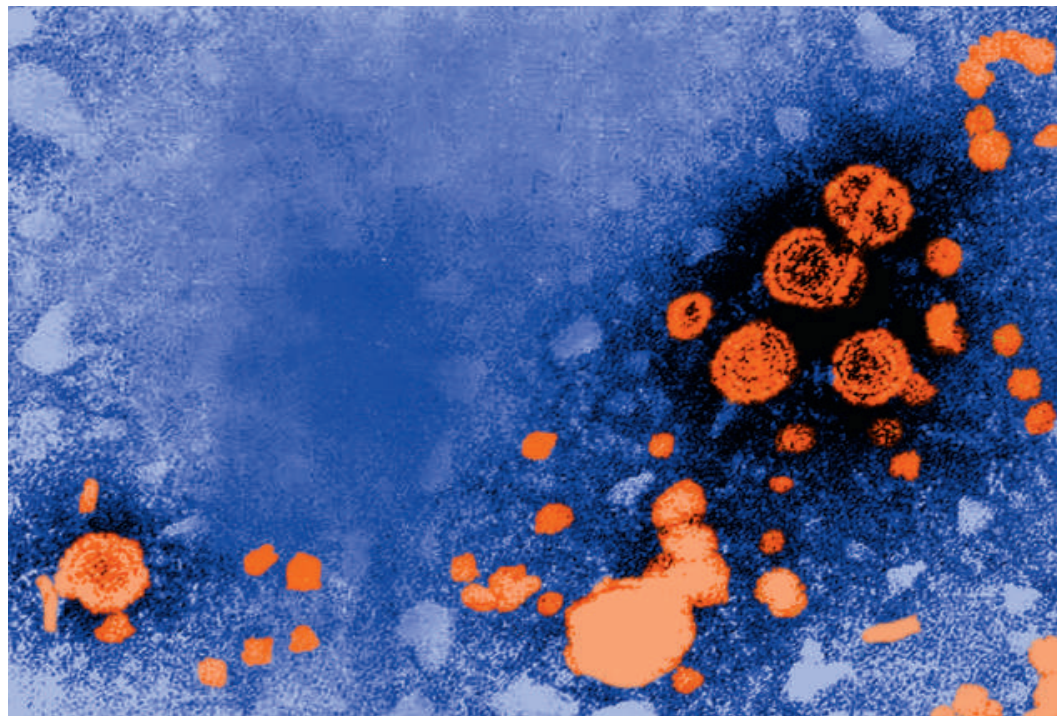

L'hépatite B (voir la figure) peut être prévenue aujourd'hui par une vaccination efficace, recommandée en Suisse à tous les adolescents.

larite, en glomérulonéphrite ou en lymphome à cellules B non hodgkinien. On a aussi rapporté des cas d'insulinorésistance, de diabète sucré et d'artériosclérose, avec en corollaire un risque accru d'événements cardiovasculaires. Les symptômes extrahépatiques les plus fréquents sont la fatigue, des difficultés de concentration et des douleurs articulaires [4].

La plupart des infections à VHC datent d'avant le début des années 90, une époque où le dépistage en routine de ces virus dans le sang n'était pas encore possible parce qu'ils n'étaient pas décelables, et où l'on ne disposait pas encore d'un vaccin efficace contre l'hépatite B. En Suisse, $60 \%$ des personnes atteintes d'hépatite $C$ se sont infectées par l'échange de seringues ou l'emploi de nécessaires d'injection contaminés par le virus [5]. D’autres causes d'infection possibles sont par exemple les transfusions sanguines administrées dans les années 80 ou une asepsie insuffisante dans la pratique de tatouages et de piercings.

L'hépatite B peut être prévenue aujourd'hui par une vaccination efficace, recommandée en Suisse à tous les adolescents. Celle-ci a permis de réduire fortement la fréquence des transmissions ces dernières années ${ }^{2}$. L'hépatite B est pratiquement incurable, mais peut comme le VIH - être contrôlée par des médicaments.

\section{On peut guérir de l'hépatite $C$}

En raison de la lente évolution de l'hépatite $\mathrm{C}$ chronique, les patients qui ont contracté l'infection avant les années 90 ne souffrent que maintenant de complications hépatiques. Une étude de l'Université de Zurich basée sur des modèles prévoit de ce fait une vague d'affections hépatiques sévères dans les années à venir [6]
On en conclut qu'il est impératif d'augmenter la couverture thérapeutique.

On dispose depuis peu de nouveaux médicaments contre l'hépatite C. Avec des taux de guérison dépassant 90\%, une durée de traitement nettement réduite et une meilleure tolérance, ces DAA (direct acting agents) représentent une véritable révolution dans le traitement de l'hépatite $\mathrm{C}$ chronique. Leur introduction a suscité une controverse portant sur leur prix élevé (les coûts vont de 30000 à 150000 francs selon la durée du traitement et l'association médicamenteuse utilisée), de sorte que l'OFSP a limité leur emploi au traitement de maladies hépatiques au moins modérément graves ou de manifestations extrahépatiques. Le recours aux traitements précédents par interféron pegylé était pareillement autolimitant: les effets indésirables étaient trop forts, un traitement pouvait durer jusqu'à une année et demie, période pendant laquelle certains patients étaient incapables de travailler. De plus, les taux de guérison étaient faibles, de l'ordre de $50 \%$. La couverture thérapeutique a pu être quasiment doublée, passant d'environ 1000-1500 traitements par an à près de 2200 en $2015^{3}$, mais cela ne suffirait pas à

Depuis 2001, les décès annuels dus à l'hépatite C en Suisse sont plus nombreux que ceux dus au VIH.

enrayer la vague d'atteintes hépatiques et de leurs complications, ni a fortiori à éliminer l'hépatite virale. Outre le rationnement de l'accès à ces traitements, l'une des raisons est sans doute que plus de la moitié des personnes touchées n'ont pas été testées. Les meilleurs traitements sont sans effet épidémiologique si la plupart des patients ignorent qu'ils sont infectés. Il est par conséquent urgent de sensibiliser le public à cette maladie.

\section{Agir maintenant}

C'est précisément le but du réseau de la Stratégie nationale de lutte contre l'hépatite. Né d'une initiative privée, ce réseau créé en 2014 réunit plus de 80 personnalités et veut développer et appliquer une stratégie de lutte contre l'hépatite pour notre pays. Tous les acteurs de l'hépatite y sont représentés: médecins spécialistes, médecins de premier recours, épidémiologistes, éthiciens, patients, agents des autorités sanitaires, politiciens et représentants de l'industrie pharmaceutique et des caisses-maladie. Leur vision commune: éliminer l'hépatite virale à l'horizon 2030. Les choses commencent aussi à bouger au niveau international: ainsi, l'Organisation Mondiale de la Santé (OMS) prépare une 
stratégie globale de lutte contre l'hépatite virale. Le projet s'est également fixé pour but de faire en sorte que l'hépatite virale cesse d'être un problème de santé publique d'ici 2030. La stratégie doit être adoptée par les Etats membres en mai de cette année [7]. L'épidémie d'hépatite virale et ses conséquences ont été sous-estimées pendant des années. Or, nous avons avec le VIH l'exemple d'une stratégie cohérente, impliquant une coordination des activités des acteurs et assortie de mesures efficaces d'information et de prise en charge, qui est appliquée avec succès de longue date et a aussi valeur de modèle à l'échelon international. Une telle stratégie serait nécessaire et possible dans le cas de l'hépatite virale. En effet, l'excellent système de santé suisse et l'efficacité de la prévention, y compris dans les groupes de population particulièrement touchés, offrent précisément la condition idéale pour que la Suisse assume rapidement un rôle précurseur en Europe dans la lutte contre l'hépatite vi-

\section{L'épidémie d'hépatite virale et ses consé- quences ont été sous-estimées pendant des années.}

rale, et puisse parvenir à réduire, voire à empêcher les conséquences menaçantes de ces infections virales. Mais pour cela, il faut une volonté politique. L'OFSP effectue cette année une analyse de la situation pour l'hépatite $\mathrm{B}$ et $\mathrm{C}$ et entend adopter des mesures appropriées au vu des résultats [8]. L'important est de ne pas perdre une année supplémentaire à attendre, mais de prendre immédiatement des mesures. La Stratégie nationale contre l'hépatite, née d'une initiative privée, poursuit à cette fin le dialogue avec les autorités sanitaires nationales et cantonales. Concrètement, le projet d'un partenariat public-privé est actuellement sur la table. La Stratégie nationale de lutte contre l'hépatite est prête à engager rapidement une collaboration soutenue et sans complications avec les autorités sani-

\section{On dispose depuis peu de nouveaux médica- ments contre l'hépatite $C$.}

taires; elle est convaincue que nous pourrons ainsi réaliser très bientôt une percée dans la lutte contre l'hépatite virale.

\section{Références}

1 Kohler P, Schmidt AJ, Cavassini M, Furrer H, Calmy A, Battegay M, Bernasconi E, Ledergerber B, Vernazza P. Swiss HIV Cohort Study (2015). The HIV care cascade in Switzerland: reaching the UNAIDS/ WHO targets for patients diagnosed with HIV. AIDS.

2 Lee M, Yang H, Lu S, Jen C, You S, Wang L, Wang C, Chen W, Chen C, R.E.V.E.A.L.-HCV Study Group (2012): Chronic hepatitis C virus infection increases mortality from hepatic and extrahepatic diseases: A community-based long-term prospective study. Journal of Investigative Democracy 206: 469-77.

3 Van der Meer A, Veldt B, Feld J, Wedemeyer H, Dufour J, Lammert F, Duarte-Rojo A, Heathcote E, Manns M, Kuske L, Zeuzem S, Hofmann W, de Knegt R, Hansen B, Janssen H. (2012): Association between sustained virological response and all-cause mortality among patients with chronic hepatitis $\mathrm{C}$ and advanced hepatic fibrosis. Journal of the American Medical Association 308(24): 2584-93.

4 Negro F, Forton D, Craxi A, Sulkowski MS, Feld JJ, Manns MP. Extrahepatic Morbidity and Mortality of Chronic Hepatitis C (2015). Gastroenterology Nov;149(6):1345-60.

5 Prasad L, Masserey-Spicher V, Zwahlen M, Rickenbach M, Helbling B, Negro F. Swiss Hepatitis C Cohort Study Group (2007): Cohort profile: The swiss hepatitis $C$ cohort study (SCCS).

6 Müllhaupt B, Bruggmann P, Bihl F, Blach S, Lavanchy D, Razavi H, Semela D, Negro F. (2015): Modeling the health and economic burden of Hepatitis C in Switzerland, PLoS One 24 10(6): e0125214.

7 Lien vers l'ébauche de projet: http://www.who.int/hiv/ strategy2016-2021/fr/

8 http://www.bag.admin.ch/evaluation/03029/index.html?lang=fr

Crédit photo

CDC / Dr Erskine Palmer

Informations complémentaires: www.hepatite-suisse.ch 